\title{
Automatic Brain Tumor Detection in T2-weighted Magnetic Resonance Images
}

\author{
P. Dvořák ${ }^{1,3}$, W.G. Kropatsch ${ }^{2}$, K. Bartušek ${ }^{3}$ \\ ${ }^{1}$ Deptartment of Telecommunications, Faculty of Electrical Engineering and Communication, Brno University of \\ Technology, Technicka 12, 61200 Brno, Czech Republic, pavel.dvorak@phd.feec.vutbr.cz \\ ${ }^{2}$ Pattern Recognition and Image Processing Group, Institute of Computer Graphics and Algorithms, Faculty of Informatics, \\ Vienna University of Technology, Favoritenstr. 9/186-3, A-1040 Vienna, Austria \\ ${ }^{3}$ Institute of Scientific Instruments of the ASCR, v.v.i., Kralovopolska 147, 61264 Brno, Czech Republic
}

\begin{abstract}
This work focuses on fully automatic detection of brain tumors. The first aim is to determine, whether the image contains a brain with a tumor, and if it does, localize it. The goal of this work is not the exact segmentation of tumors, but the localization of their approximate position. The test database contains $203 \mathrm{~T} 2$-weighted images of which 131 are images of healthy brain and the remaining 72 images contain brain with pathological area. The estimation, whether the image shows an afflicted brain and where a pathological area is, is done by multi resolution symmetry analysis. The first goal was tested by five-fold cross-validation technique with 100 repetitions to avoid the result dependency on sample order. This part of the proposed method reaches the true positive rate of $87.52 \%$ and the true negative rate of $\mathbf{9 3 . 1 4 \%}$ for an afflicted brain detection. The evaluation of the second part of the algorithm was carried out by comparing the estimated location to the true tumor location. The detection of the tumor location reaches the rate of $95.83 \%$ of correct anomaly detection and the rate $87.5 \%$ of correct tumor location.
\end{abstract}

Keywords: Brain tumor, brain tumor detection, symmetry analysis.

\section{INTRODUCTION}

$\mathrm{T}$ HIS WORK focuses on automatic brain tumor detection in stand-alone 2D T2-weighted images of human brain and extends our previous work published in [1]. The aim of the method proposed in the previous paper was the detection of a tumor in brain images. The assumption for an input was an image containing a tumor, so it did not deal with detection of images containing a brain with a tumor. The method proposed in the current work extends the previous one, because it can filter out images of healthy brain.

The detection of brain tumors is generally a more complex task than the detection of any other image object. Pattern recognition usually relies on the shape of the required objects. Since the tumor shape varies in each case, other properties have to be used. The general properties of healthy brain are widely used as a prior-knowledge. One of them is the probability of tissue locations using the probabilistic brain atlas, which is used, e.g., in [2]. Another widely used knowledge, which is used in this article, is the approximate left-right symmetry of healthy brain. This approach is also used, e.g., in [3] [4] [5]. The areas breaking this symmetry are most likely parts of a tumor.

There are also many other methods used for tumor extraction, but they usually rely on machine learning algorithms such as Support Vector Machine [6]. For this purpose, many algorithms need to have patient-specific training datasets. This makes the method more demanding for the experts. These methods usually rely on other contrast images, such as T1-weighted contrast enhanced images [7]. Fully automatic exact segmentation of the tumor is still an unsolved problem, as is the accurate image segmentation itself. The method proposed in this paper analyzes 2D T2-weighted magnetic resonance images and is fully automatic. It could be used for the decision, whether the tumor is present and the approximate detection of the brain tumor location for subsequent segmentation, which will be the aim of future work.

The great advantage of the symmetry approach is that the process does not need any intensity normalization, human work, etc. Another advantage is its independence on the type of the tumor. It can correctly detect anomalies in images containing a tumor, a tumor with an edema or only an edema, which is an abnormal accumulation of the fluid around the tumor and is present only with particular types of tumors.

\section{Proposed Method}

The principle of the proposed method is based on our previous work described in [1]. That method was based on multi resolution symmetry analysis. It detected the most asymmetric regions and only those were considered in further computation. This was repeated three times for different size of the checked region.

The input of the whole process is a stand-alone 2D T2weighted magnetic resonance image. No neighbor slices are considered. The reason for T2-weighted image is the good visibility of tumors in this type of image.

The tumor detection process consists of several steps. The flow chart can be seen in Fig. 1. The first step is the skull extraction followed by image cutting. For this cut image, the probabilistic map of anomalies is computed, and features are extracted from this map. These features are used for the de- 


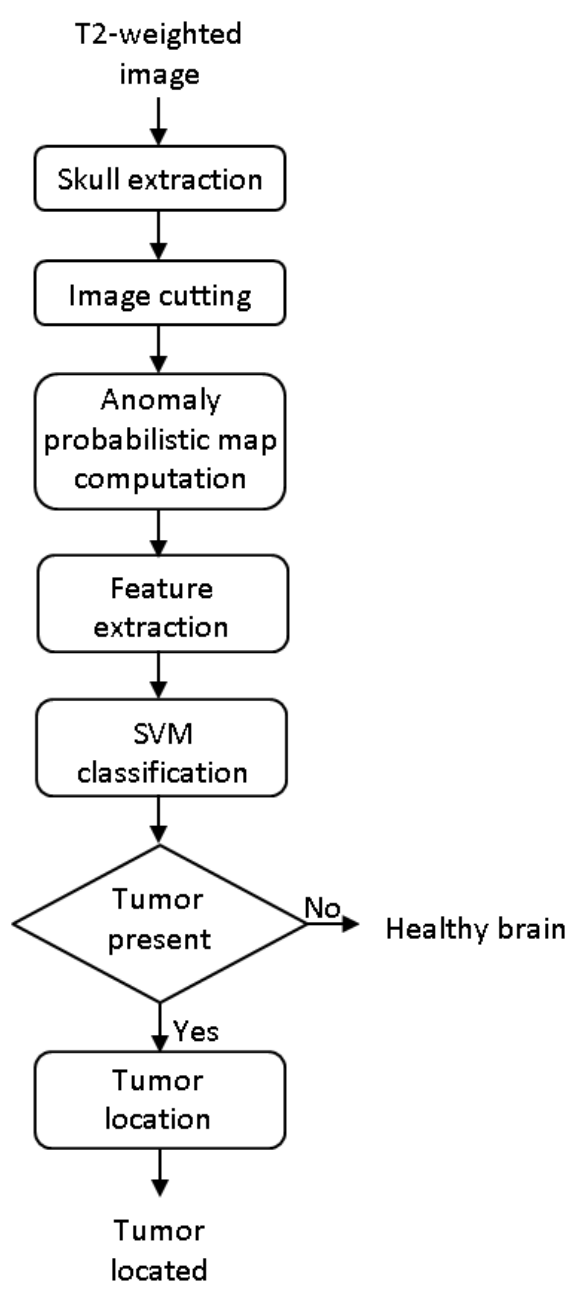

Fig. 1: Flow chart of proposed method.

cision, whether a pathological area is present in the image. If so, this area is located and then the decision, which half contains the tumor, is made. If no pathological area is present, the image shows a healthy brain and no other computation is needed. The detection of the symmetry axis is skipped because the input data were aligned in previous processing. For the purpose of detecting the symmetry axis, the existing algorithms described in [8] or [9] can be used. One of the aims of the future work is addition of such method as a preprocessing step.

\subsection{Skull extraction}

The extraction of the skull is based on a technique mentioned in [10] called Active contour, or Snakes [11]. At first, the smallest rectangle surrounding the skull, whose sides are parallel to the image sides, is detected. The initial mask is set to this rectangle to be sure that the whole skull is inside the mask. Then the algorithm is executed.

Assuming that the head is approximately symmetric, the symmetry axis is set to be parallel to the vertical axis and to divide the detected rectangle into two parts of the same size.

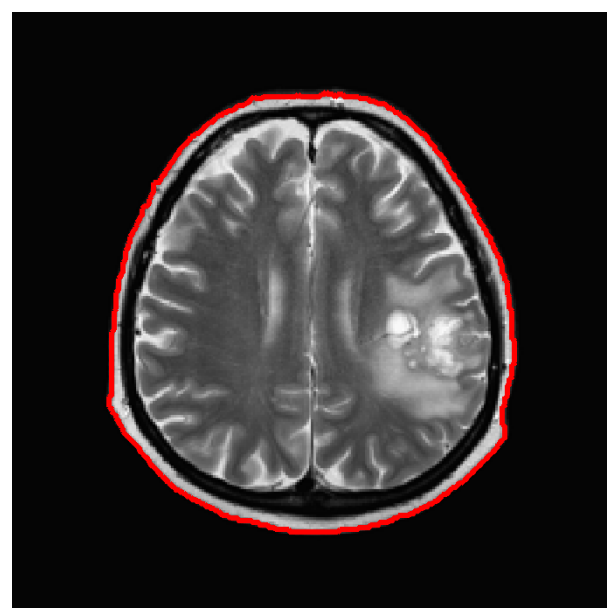

Fig. 2: Skull extraction.

The result of the segmentation algorithm is a set of contours. The outer contour is considered to be the skull contour. The holes inside this region are filled and the resulting mask is applied to the input image. The result of the skull extraction is shown in Fig. 2.

\subsection{Symmetry checking}

The most important part of this work is the detection of symmetry anomalies, which are usually caused by a brain tumor, whose detection is the main purpose of this article. The first step of this process is dividing the input image into two approximately symmetric halves.

Assuming that the head is not rotated and the skull is approximately symmetric, the symmetry axis is parallel to the vertical axis and divides the image of the detected brain into two parts of the same size. Since the method is not pixelbased, the precision of the determined symmetry axis has not significant influence.

Since the features are extracted from the computed asymmetries, the size of the image has to be normalized. Hence, every cropped image is resized into the same size, concretely 256x256 pixels.

A squared block, with the side length computed as one quarter of the cropped image side length, is created. This size and sizes computed in the following computation are suitable for the detection of both small and large tumors. The algorithm goes through both halves symmetrically by this block. The step size is smaller than the block size to ensure the overlapping of particular areas. These areas are compared with their opposite symmetric part. In this case, the step size of one eighth of the block size was set.

Comparison is done by the Bhattacharya Coefficient [12]. Normalized histograms with the same range are computed from both parts and the Bhattacharya Coefficient (BC) is computed from these histograms as follows [12]:

$$
B C=\sum_{i=1}^{N} \sqrt{l(i) \cdot r(i)}
$$






(a)

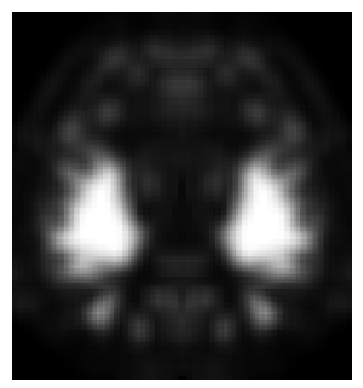

(c)

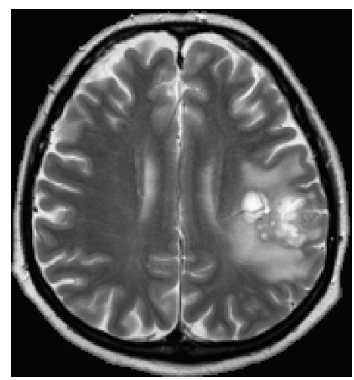

(e)

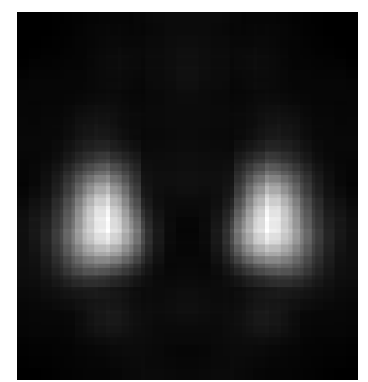

(b)

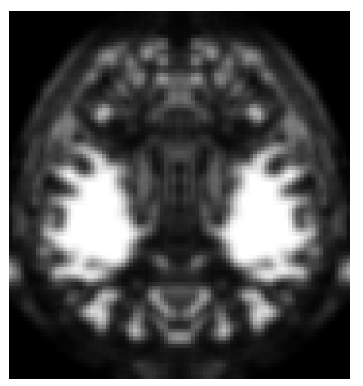

(d)

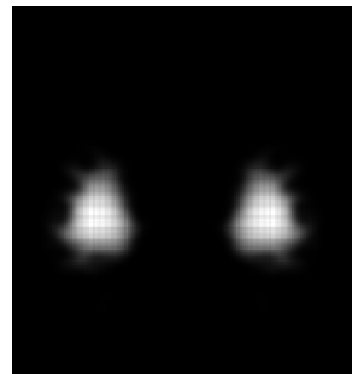

(f)
Fig. 3: Asymmetry probabilistic maps for block side length equal to $1 / 4,1 / 8,1 / 16$, and 1/32 of shorter side of cut image are shown in (a), (b), (c), and (d), respectively. In (e) and (f), input image and total probabilistic map are shown, respectively.

where $N$ denotes the number of bins in the histogram, $l$ and $r$ denote histograms of blocks in the left and the right half, respectively.

The range of values of $\mathrm{BC}$ is $\langle 0,1\rangle$, where the smaller the value, the bigger the difference between histograms. For the next computation, the asymmetry is computed as:

$$
A=1-B C \text {. }
$$

This asymmetry is computed for all blocks. Since the step size is smaller than the block size, the overlap exists and more values of asymmetry are present for most pixels. To obtain the appropriate asymmetry map, the mean of all values computed for a particular pixel is computed.

The computed values of asymmetry create the asymmetry map, which expresses the probability of tumor presence in a particular location. The higher the asymmetry is, the higher is the probability of the tumor presence in a given location.

\subsection{Multi-resolution probabilistic map computation}

The whole cycle of symmetry checking is repeated four times but with different size of block. Height and width of the block are iteratively reduced to the half of the previous value. So the size of the block is $1 / 1,1 / 4,1 / 16$, and $1 / 64$ of the initial size, respectively. The purpose of smaller areas is the more precise detection of asymmetry. This approach corresponds to the multi resolution image analysis described in [13]. A block size of $1 / 256$ of the initial size was tested as well, but the results were not improved and the maximum of asymmetry coefficient for this block size was equal to 1 for every image in database.

The output of each cycle is a probabilistic map of anomalies. The product of values corresponding to a particular pixel is computed. The output is the new multi resolution probabilistic map.

The examples of particular probabilistic maps are shown in Fig. 3.

\subsection{Feature extraction}

In the next step, features are extracted from computed probabilistic maps. These features are used for the decision, whether the particular image contains a pathological area. According to experiments, the relative and absolute thresholding can help in distinguishing between images of healthy and afflicted brain. The thresholding creates a given number of regions with a given size, and both of these values differ for healthy and afflicted brains. The extracted features are as follows:

- global maximum of the total probabilistic map,

- maximum of each probabilistic map for a particular block size,

- number of regions created by absolute value thresholding the total probabilistic map and the sum of their size,

- number of regions created by relative value thresholding the total probabilistic map and the sum of their size.

Global maximum of the total probabilistic map: Since the proposed method is based on searching the pathological area by symmetry checking, the maximum of asymmetry coefficient is the main feature, which can be used for classifying the image.

Maximum of each probabilistic map for a particular block size: Other usable features are maxima of each probabilistic map computed in the previous step. Functional dependency of the anomaly coefficient on the block size is non-ascending, it means that for a smaller block, the anomaly coefficient is greater or equal to that of a larger block.

For images with large tumors, this value is high even for a large block, while for small tumors, this function reaches the maximum later. For healthy brains, this function is even more shifted. 


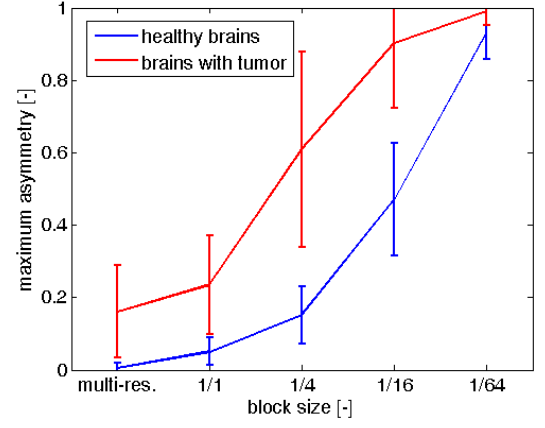

Fig. 4: The maximum asymmetry for multi-resolution asymmetry map and for particular block sizes. Blue: healthy brains, Reg: afflicted brains.

The maximum asymmetry for multi-resolution asymmetry map and for particular block sizes are shown in Fig. 4.

Number of regions created by absolute value thresholding the total probabilistic map and the sum of their size: This feature assumes that the anomaly probabilistic map of healthy brain contains a smaller value compared to the brain with tumor. When the thresholding is done, in case of healthy brain, the result is a smaller number of regions and also a smaller sum of their size. In most healthy cases, both numbers are equal to zero.

Number of regions created by relative value thresholding the total probabilistic map and the sum of their size: For the extraction of this feature, the total probabilistic map is thresholded by relative value computed from the maximum of this map. Here, it is assumed that for brain with tumor, there is a significant peak in the part where a tumor is situated. So for thresholding by a value computed from this maximum, healthy areas are filtered out, because they are usually much more symmetric. Moreover, the tumor is in most cases concentrated in one location, therefore a small number of regions is created by thresholding.

In case of a healthy brain, the situation is inverse. The maximum is comparable to values in other parts, so more regions are created by thresholding, moreover, they are spread into the whole brain. For large tumors, the sum of areas is comparable to the one of healthy brain, but the number of regions is smaller.

For both relative and absolute thresholding, 10 different levels of threshold are set, so 10 values are extracted for each feature. Statistical graphs of number of region and sum of their size for both relative and absolute thresholding for different threshold levels are shown in Fig. 5.

\subsection{Tumor detection}

The part of the proposed method, which detects the presence of a pathological area in a particular brain, uses a supervised machine learning algorithm Support Vector Machine (SVM), invented by Cortes and Vapnik in 1995 [14], with linear kernel function. The features described in the previous section are used for image classification. Since an existing technique

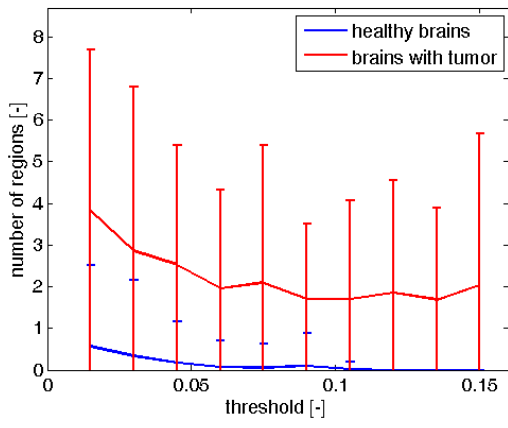

(a)

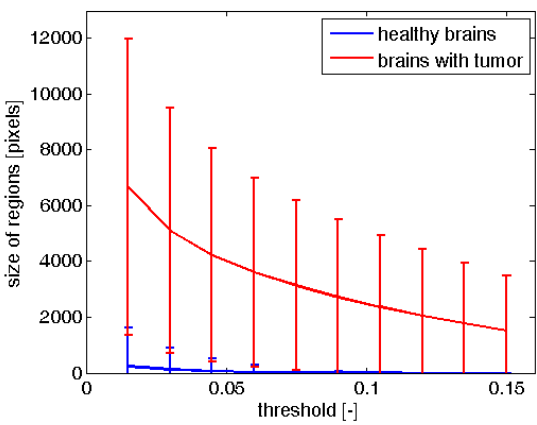

(b)

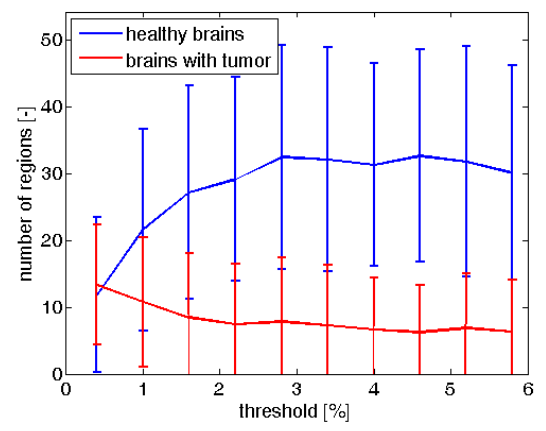

(c)

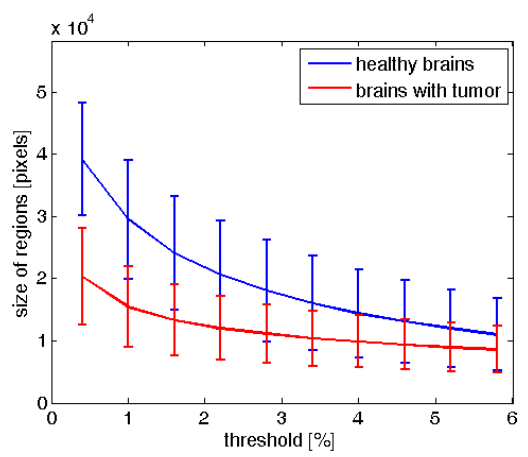

(d)

Fig. 5: (a) and (b) show number of regions and the sum of their sizes for different absolute threshold levels. (c) and (d) show number of regions and the sum of their sizes for different relative threshold levels to maximum in a particular image. In all graphs, comparison of mean value with standard deviation for healthy (blue) and afflicted (red) brains is shown. 
described in many literatures is used here, the reader can find more information, e.g., in [6].

\subsection{Locating the tumor}

If the image is labeled as an image containing a brain with tumor, the location of the tumor is searched. For this asymmetry detection is used and it is based on the principle described in [1]. Since an accurate segmentation is not the aim of the algorithm and the output will be used as a feature for further segmentation, the probabilistic map is considered to be the proper output. On the other hand, the determination of approximate location of the tumor is interesting as a demonstrating result and is also needed for decision on which side the tumor is. The approximate location is determined by thresholding the probabilistic map. The threshold is computed as an average value of the maxima of the probabilistic map of the healthy brain. If the tumor size is small or it is not clearly visible, the maximum of the probabilistic map is lower than the threshold. In that case, no region is extracted. If this happens, the relative thresholding, where the threshold is computed as $5 \%$ of the maximal value, is done.

Since more regions can be a result of the thresholding, the one where the maximum is located, is extracted.

After the region detection, the decision, in which of these 2 regions the tumor is, has to be done. In [1], two possibilities were tested. The one that is also used in [10] reached slightly better result and it simplifies the brain extraction to the skull extraction. Thus, comparing the average intensity of these regions is used for a final decision. Since tumors in T2weighted images produce stronger signal than white matter, in which they are located in most cases, the region with higher average intensity is labeled as the one containing the tumor.

For demonstration, the maximum value of probabilistic map of tumor location and the approximate region determined as a tumor created by thresholding are shown in Fig. 6 .

\section{TESTING}

The test database consists of 203 T2-weighted images of brain with various image sizes. The smallest image has the size of $256 \times 256 \mathrm{px}$, while the largest one has the size of $630 \times 630$ px. Since the cropped images are resized into the same size of $256 \times 256 \mathrm{px}$, the image size does not matter. 131 of test images are the images of healthy brain from 11 patients. 72 images from 13 patients are the images of brain containing a tumor, a tumor with an edema or only an edema. These images are of various resolutions and contain pathological areas of various shape, size and location. In the database, there are images of 12 small tumors, whose size is less than $2 \%$ of the skull size, 30 medium tumors, whose size is between 2 and $9 \%$ of the skull size, and 30 large tumors, whose size is more than $10 \%$.

At first, the detection of a pathological area presence in the particular image was tested by a five-fold cross-validation process. It means that the samples in the database were randomly ordered and split into five groups. In five cycles, each

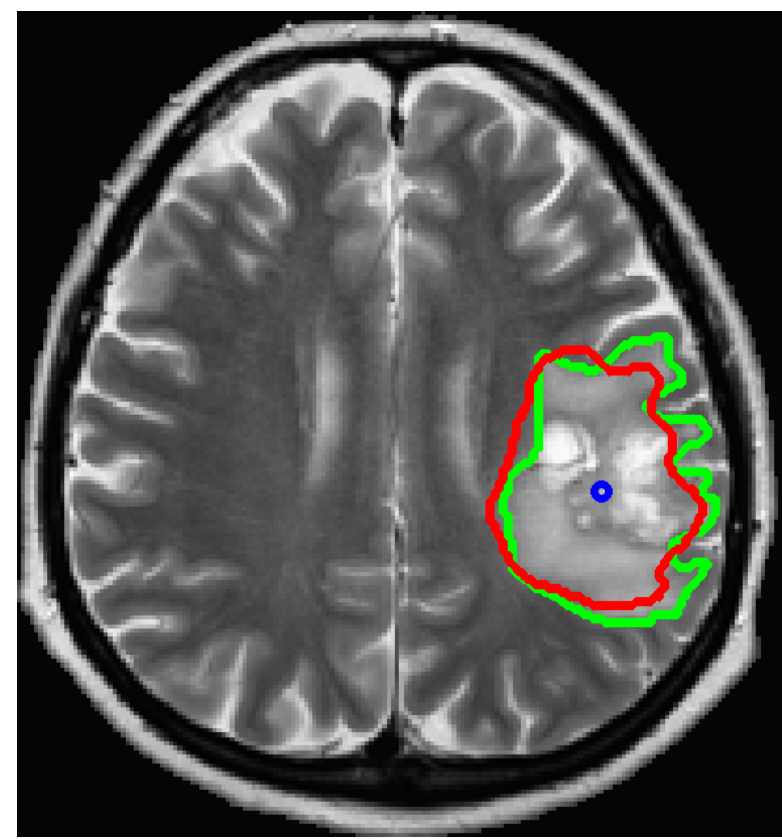

Fig. 6: The maximum value of the tumor probability (blue circle) and the computed approximate borders of the tumor (red curve) compared to the ground truth (green curve).

of these groups was once used as the validation set, while the remaining four sets served as training data.

To avoid dependency on the order of samples, the cycle of random ordering and five-fold cross-validation was repeated one hundred times.

After the decision, whether the image contains a tumor is done, the tumor location is found. This part of the system was tested for all 72 images in the database containing a brain with tumor. Since the probabilistic map is symmetric, there are two global maxima in the map. One of them is situated on the left, while the other on the right side. At first, the location of one of them compared to true tumor location will be evaluated. After that, the determination of the approximate tumor location and the decision, on which side the tumor is, will be evaluated.

The determination of the position and structure of the tumor is evaluated by the Jaccard index [15]. This index computes the similarity of two sets and is computed as:

$$
J(A, B)=\frac{A \cap B}{A \cup B} .
$$

\section{RESULTS}

\subsection{Tumor detection}

In Tab. 1, the overall results of tumor presence detection are shown. The test results are in rows, while columns express the ground truth. The presence of tumor was correctly detected in $87.52 \%$ of cases and the absence of it was correctly classified in $93.14 \%$. It means that $6.86 \%$ of the images of healthy brain were classified as images where the tumor is present, and $12.48 \%$ of images containing a tumor were classified as healthy. 


\begin{tabular}{l|lc}
\hline \hline & Tumor present & Tumor absent \\
\hline Test positive & $87.52 \%$ & $6.86 \%$ \\
Test negative & $12.48 \%$ & $93.14 \%$ \\
\hline \hline
\end{tabular}

Table 1: Tumor detection performance.

\begin{tabular}{l|ccc}
\hline \hline Tumor size & Small & Medium & \multicolumn{1}{c}{ Large } \\
\hline Test positive & $52.08 \%$ & $91.37 \%$ & $99.00 \%$ \\
Test negative & $47.92 \%$ & $8.63 \%$ & $1.00 \%$ \\
\hline \hline
\end{tabular}

Table 2: Tumor detection performance for different tumor sizes.

Since the number of images of healthy brain is not much higher than the number of images of afflicted brain, the overall accuracy of the algorithm can be also expressed. The overall accuracy of this part of the proposed system is $91.15 \%$.

Most cases of false negative were images where only an edema or a tumor of small size was present, as can be seen in Tab. 2. The performance for these cases could be improved by combining neighbor slices. Most cases of the false positive results were the images containing an unexpected symmetric anomaly as irregular shape of a brain or a skull.

In Tab. 3, the summary of the number of correct classifications for a particular image can be seen. 59 images of afflicted brain were correctly classified in all tests, but on the other hand, there are five images of small tumor and one image of medium tumor that were misclassified in almost every test.

\subsection{Tumor location}

The detection of tumor location was tested by comparing the maximum of probabilistic map and the true location of the tumor. The performance was tested for all images with tumor in the database, even those that were not detected in the previous step. The overall performance will be evaluated in the next section.

In 69 cases, one of the maxima of probabilistic map was situated inside the true tumor location. In the remaining three cases, both maxima were outside the region. In all incorrect detections, only an edema was present and it was hardly visible even for human experts.

Although the accurate segmentation is not the aim of this work, the approximate structure detection could be evaluated. In 6 of these 69 cases, where the anomaly was correctly detected, the opposite side was chosen as the one with tumor. In most cases, this happened for images with atypical teratoid rhabdoid tumor, which breaks the assumption of stronger signal in pathological area. The approximate structure was correctly determined in 37 cases. In the remaining cases, a smaller region was extracted or other parts were included. This could be caused by an anomaly created around the tumor caused by its pressure to the surrounding tissues.

The results of the tumor structure determination are shown in Tab. 4. The Jaccard index expresses similarity of two sets.

\begin{tabular}{l|ccc}
\hline \hline & \multicolumn{3}{|c}{ Tumor size } \\
Correct classification [\%] & Small & Medium & Large \\
\hline 100 & 4 & 24 & 27 \\
$90-99$ & 1 & 2 & 1 \\
$50-90$ & 0 & 2 & 2 \\
$20-50$ & 2 & 1 & 0 \\
$<20$ & 5 & 1 & 0 \\
\hline \hline
\end{tabular}

Table 3: Number of correct classifications for a particular image.

\begin{tabular}{l|ccc}
\hline \hline & \multicolumn{3}{|c}{ Tumor size } \\
Jaccard index & Small & Medium & Large \\
\hline $0.7-0.9$ & 0 & 7 & 19 \\
$0.5-0.7$ & 2 & 11 & 10 \\
$0.3-0.5$ & 3 & 5 & 0 \\
$0.1-0.3$ & 4 & 2 & 0 \\
$0.01-0.1$ & 0 & 1 & 0 \\
0 (opposite side) & 2 & 3 & 1 \\
0 (incorrect anomaly det.) & 1 & 1 & 0 \\
\hline \hline
\end{tabular}

Table 4: Jaccard index for tumor extraction results.

The range of the Jaccard index is $\langle 0 ; 1\rangle$, where $J=1$ expresses the complete agreement. Since it is very hard to achieve this result and it has not been achieved in this work, this value is not stated in the table. The comparison between tumors of different sizes can be seen. The results with value equal to zero can be caused by the extraction of a region without any part of a tumor or by the incorrect side determination as can be seen in the two bottom lines of the table. For the tumors of medium and large size, the structure determination reaches fairly good results. The results for most intervals of the Jaccard index are shown in Fig. 7

\subsection{Overall results}

62 images of afflicted brain were correctly classified in all tests during the cross validation cycle with 100 repetitions. Four images were misclassified in 1-10 tests, four images in 11-50 tests, three images in 51-80 tests and six images in more than 80 tests.

Two of the images, where the maximum of probabilistic map was situated outside the tumor region, were also misclassified as a healthy brain in more than 90 tests. Both of these images contained only hardly visible edema. Another image, where the symmetric anomaly was correctly detected, but the tumor was located on the opposite side, was misclassified in more than 50 tests.

In 64 of 65 cases that were correctly classified as an afflicted brain in most cases, one of the maximum of probabilistic map of particular image was situated inside the tumor area. In 60 cases, the side where the tumor was, was correctly found. The approximate structure of the tumor was correctly determined in 36 of correctly classified cases. 


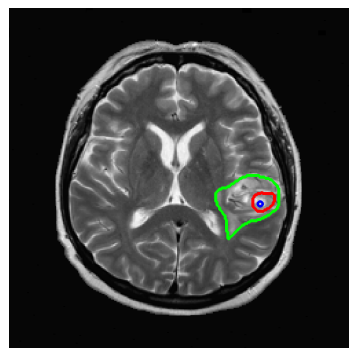

(a)

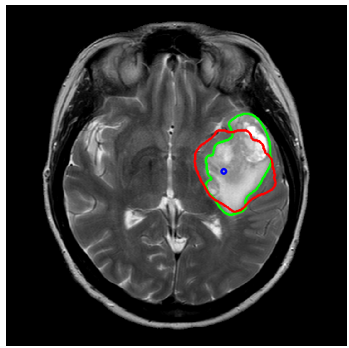

(c)

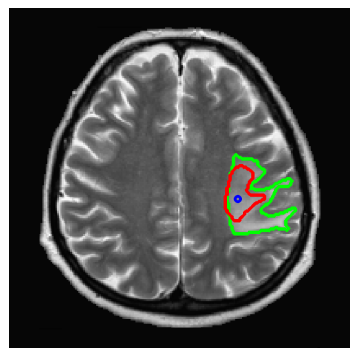

(b)

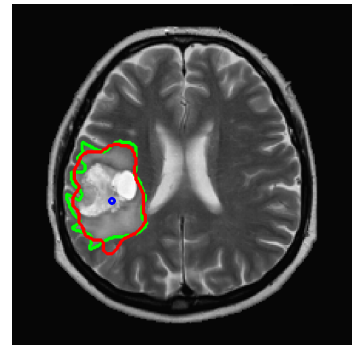

(d)
Fig. 7: The results of the tumor area extraction for different values of the resulting Jaccard index. (a) $\mathrm{J}=0.13$, (b) $\mathrm{J}=0.38$, (c) $\mathrm{J}=0.64$, (d) $\mathrm{J}=0.83$. The maximum value of the tumor probability (blue circle) and the computed approximate borders of the tumor (red curve) compared to the ground truth (green curve).

\section{DISCUSSION / CONCLUSION}

The aim of this work was the detection of images containing an abnormality caused by tumor and detection of the approximate location. The evaluation was divided into 2 parts: detection of image containing a tumor and detection of tumor location. The image with tumor detection reaches the true positive rate of $87.52 \%$ and the true negative rate of $93.14 \%$. The detection of the tumor location reaches the rate of $95.83 \%$ of correct anomaly detection and $87.5 \%$ of correct tumor location. Compared to the previous method [1], it can also more precisely capture the topological structure of the detected tumor. That method reached results of $98.63 \%$ of the detection of main part of the tumor, $75.34 \%$ of the correct anomaly detection, and $71.23 \%$ of the correct tumor extraction. Current work improves the performance and extends the previous one by automatic decision, whether a pathological area is present, and the more precise structure estimation.

The proposed method can be also used for detection of tumor in 3D axial, but particular axial slices would be evaluated separately. Hence, the attention in the future work will also be paid to the relations between neighbor slices and after that, the work will continue with extending the proposed algorithm to $3 \mathrm{D}$.

The future work will also include implementation of the automatic symmetry axis detection, based on literature referred to in 2 , and the more precise extraction of the tumor based on current results.

\section{ACKNOWLEDGEMENT}

This research work is funded by project SIX CZ.1.05/2.1.00/03.0072, EU ECOP EE.2.3.20.0094, project GACR 102/12/1104, and project CZ.1.05/2.1.00/01.0017 (ED0017/01/01), Czech Republic.

\section{REFERENCES}

[1] Dvorak, P., Kropatsch, W.G., Bartusek, K. (2013). Pathological area detection in MR images of brain. Elektrorevue, 4(1), 17-21.

[2] Cuadra, M.B., Pollo, C., Bardera, A., Cuisenaire, O., Villemure, J.G., Thiran, J.P. (2004). Atlas-based segmentation of pathological MR brain images using a model of lesion growth. IEEE Transactions on Medical Imaging, 23(1), 1301-1314.

[3] Cap, M., Marcon, P., Gescheidtova, E., Bartusek, K. (2013). Automatic detection and segmentation of the tumor tissue. In Proceedings of PIERS 2013, Taipei, 5356.

[4] Pedoia, V., Binaghi, E., Balbi, S., De Benedictis, A., Monti, E., Minotto, R. (2012). Glial brain tumor detection by using symmetry analysis. In Proceedings of SPIE, Volume 8314, Medical Imaging 2012: Image Processing, 831445.

[5] Somasundaram, K., Kalaiselvi, T., (2010). Automatic detection of brain tumor from MRI scans using maxima transform. In National Conference on Image Processing (NCIMP).

[6] Mikulka, J., Gescheidtova, E. (2013). An improved segmentation of brain tumor, edema and necrosis. In Proceedings of PIERS 2013, Taipei, 25-28.

[7] Capelle, A.S., Color, O., Fernandez-Maloigne, C. (2004). Evidential segmentation scheme of multi-echo MR images for the detection of brain tumors using neighborhood information. Information Fusion, 5, 103 216.

[8] Karuppanagounder, S., Thiruvenkadam, K. (2009). A novel technique for finding the boundary between the cerebral hemispheres from MR axial head scans. In Proceedings of the 4th Indian International Conference on Artificial Intelligence, IICAI 2009 Tumkur, Karnataka, India, December 16-18, 1486-1502.

[9] Ruppert, G.C.S., Teverovskiy, L., Yu, C.-P., Falcao, A.X., Liu, Y. (2011). A new symmetry-based method for mid-sagittal plane extraction in neuroimages. In IEEE International Symposium on Biomedical Imaging: From Macro to Nano.

[10] Ray, N., Saha, B.N., Graham Brown, M.R. (2007). Locating brain tumors from MR imagery using symmetry. In The Forty-First Asilomar Conference on Signals, Systems and Computers, ACSSC 2007, 224-228.

[11] Mikulka, J., Gescheidtova, E., Bartusek, K. (2012). Soft-tissues image processing: Comparison of tradi- 
tional segmentation methods with 2D active contour methods. Measurement Science Review, 12(4), 153-161.

[12] Bhattacharyya, A. (1943). On a measure of divergence between two statistical populations defined by their probability distribution. In Bulletin of the Calcutta Mathematical Society,35, 99-110.

[13] Kropatsch, W.G., Haxhimusa, Y., Ion, A. (2007). Multiresolution image segmentations in graph pyramids. In Applied Graph Theory in Computer Vision and Pattern Recognition Studies in Computational Intelligence, 52,
$3-41$.

[14] Cortes, C., Vapnik, V.N. (1995). Support-Vector Networks. Machine Learning, 20(3), 271-297.

[15] Jaccard, P. (1908). Nouvelles recherches sur la distribution florale. Bulletin de la Société Vaudoise des Sciences Naturelles, 44, 223-270.

Received June 3, 2013. Accepted October 15, 2013. 